\title{
Pregnant women in UK are offered whooping cough vaccine to protect newborns
}

In the first paragraph of this News story we incorrectly referred to whooping cough as a "virus" (BMJ 2012;345:e6594, doi:10. 1136/bmj.e6594). As pointed out to us by readers, whooping Cite this as: BMJ 2012;345:e6806 cough is not a virus, but a bacterial disease. 